\title{
Competence in pediatric urology upon graduation from residency: perceptions of residents, program directors and pediatric urologists
}

\author{
Jennifer J. Mickelson, MD, FRCSC; ${ }^{*}$ Andrew E. MacNeily, MD, FRCSC, FAAP; ${ }^{\dagger}$ Dinesh Samarasekera, MD; ; \\ Darren Beiko, MD, FRCSC,; Kourosh Afshar, MD, MHSc, FRCSC, FAAP ${ }^{+}$
}

See related article on page 211

\section{Abstract}

Objective: We aimed to clarify the scope of pediatric urological procedures that Canadian urology residents are perceived to be competent to perform upon graduation.

Methods: We conducted a survey from April 2005 to June 2006 of urology residency program directors (UPDs), senior urology residents (SURs) and Pediatric Urologists of Canada (PUC) members from all 12 Canadian training programs. Questions focused on which of 23 pediatric urological procedures the 3 study groups perceived urology residents would be competent to perform upon completion of residency without further fellowship training. Procedures were based on the "A," "B" and "C" lists of procedures (least complex to most complex) as outlined in the Royal College of Physicians and Surgeons of Canada Objectives of Training in Urology.

Results: Response rates were 12/12 (100\%), 41/53 (77\%) and 17/23 (74\%) for UPDs, SURs and PUC members, respectively. Average exposure to pediatric urology during residency was 5.4 (range 3-9) months and considered sufficient by $75 \%$ of UPDs and $69 \%$ of SURs, but only $41 \%$ of PUC members $(p=0.05)$. Overall, the 3 groups disagreed on the level of competence for performing level " $A$ " and "B" procedures, with significant disagreement between PUC members and UPDs as well as SURs $(p<0.005)$.

Conclusion: PUC members perceive Canadian urology residents' exposure to pediatric urology as insufficient and their competence for procedures of low to moderate complexity as inadequate. Further investigation regarding exposure to and competence in other emerging subspecialty spheres of urology may be warranted. Ongoing assessment of the objectives for training in pediatric urology is required.

CUAJ 2008;2(3):205-10

\section{Introduction}

Pediatric urology is the first sphere of our specialty to be granted the opportunity to offer urologists a certificate of added qualification (CAQ). ${ }^{1}$ Acquisition of this CAQ will require evidence of advanced postresidency fellowship training, a focus on pediatric urology in one's scope of practice and successful completion of an examination process administered by the American Board of Urology. ${ }^{2}$ Despite this controversial move toward subspecialization and possible fragmentation within urology, exposure to pediatric urology remains an important and mandatory component of urological residency training in North America. Our goal was to assess the perceived competence of Canadian urology residents in performing a set of pediatric urological procedures upon graduation. More specifically, we sought to assess which pediatric urological procedures Canadian residents, program directors and practising pediatric urologists felt graduates should be competent to perform, and which they would actually perform, without further fellowship training.

\section{Methods}

We derived a listing of 23 pediatric urological procedures from the Royal College of Physicians and Surgeons of Canada (RCPSC) Objectives of Training in Urology (Box 1). ${ }^{3}$ These objectives are set and periodically revised by the urology specialty committee members. Briefly, procedural objectives are categorized into an " $\mathrm{A}$," "B" or " $\mathrm{C}$ " category according to levels of complexity (least to most). Category "A" procedures are those in which

all residents must be competent to independently perform .... be able to manage a patient prior to, during and after ... [and] be able to describe the management of the common complications ...

\section{Category "B" procedures}

are those that the resident will know how to do, including indications. ... the resident may not have actually done one of these procedures independently during the residency training program.

\section{Category "C" procedures}

are those for which the resident will be able to 
describe the principles of the procedure, indications for referral for the procedure and particular perioperative problems that might be encountered. ${ }^{3}$

We developed a survey pertaining to length of training in pediatric urology during residency, and a series of questions requiring binary yes/no responses regarding perceived competence for the 23 procedures listed (Appendix 1). As well, respondents were queried regarding the perceived probability of urology residents actually performing these procedures upon graduation. The final survey was assessed for face validity on a group of 15 urology residents from the University of British Columbia. A Web- and mail-based survey was carried out from April 2005 to June 2006 on 3 groups of individuals: all Canadian urology residency program directors (UPDs), 2 consecutive graduating classes of senior urology residents (SURs) in their final 6 months of training and active members of Pediatric Urologists of Canada (PUC) who were practising in a centre with a urology residency training program. Overall, the sample comprised 12 UPDs, 53 SURs and 23 PUC members. Each potential respondent received the survey with a cover letter explaining the nature of the study, its potential usefulness and the fact that the procedures in question pertained specifically to the pediatric patient. A second email reminder was sent out 2 months later. All responses were anonymous with respect to individual and institutional identity.

Data were collected in an Excel (Microsoft Corp., Redmond, Wash.) spreadsheet. All of the binary answers were first analyzed collectively, followed by subgroup analysis according to RCPSC categories. The Cochran-Mantel-Haenszel $\chi^{2}$ test was used to compare the answers in the 3 groups. The Bonferroni correction was used to adjust for multiple comparisons.

\section{Results}

A total of $70 / 88(80 \%)$ of surveys were returned. Included were 12 (100\%) from UPDs, 41 (77\%) from SURs and 17 (74\%) from PUC members. At the time of the survey, 3 PUC members were also program directors. We analyzed their responses 3 ways: with the 3 respondents' data in both the PUC and UPD data pools, only in the PUC data pool and only in the UPD data pool. A sensitivity analysis revealed no significant difference in the results. We ultimately reported these 3 respondents with the UPD data pool since it was the smallest data set.

\begin{tabular}{ll} 
Box 1: List of $\mathbf{2 3}$ procedures by category as outlined in the Royal \\
College of Physicians and Surgeons of Canada Objectives of \\
Training in Urology \\
A List & \multicolumn{1}{c}{ B List } \\
Circumcision & Nonpalpable orchiopexy \\
Incarcerated hernia & Laparoscopy for nonpalpable testis \\
Exploration of testicular torsion & Distal shaft hypospadias repair \\
Infant hernia/hydrocele repair & Cutaneous ureterostomy \\
Palpable orchiopexy & Vesicostomy \\
Meatoplasty & TUR of posterior urethral valves \\
Meatal repair for balanic hypospadias & Renal transplant \\
Infant heminephrectomy & \\
Infant pyeloplasty & \\
Ureteral reimplant & Proximal hypospadias repair \\
Insertion of suprapubic tube & Exstrophy repair \\
Augmentation cystoplasty & \\
Pediatric continent diversion & \\
Endourologic procedure for urolithiasis & \\
TUR = transurethral resection. & \\
&
\end{tabular}


The exposure of urology residents to pediatric urology averaged 5.4 (range 3-9) months during their 5 years of training. All programs had pediatric rotations in the senior years ( $\mathrm{R} 4$ or $\mathrm{R} 5$ ). Programs were evenly divided between those that provided pediatric rotations only during senior years and those that divided 2 or more rotations between the junior and senior years. There was significant disagreement among the 3 groups of respondents regarding the adequacy of the total duration of this exposure in their own particular training program (Fig. 1).

Program directors and residents consistently rated trainee competence higher than PUC members did for the " $\mathrm{A}$ " and " $\mathrm{B}$ " category of procedures, with no differences regarding the 2 " $\mathrm{C}$ " category

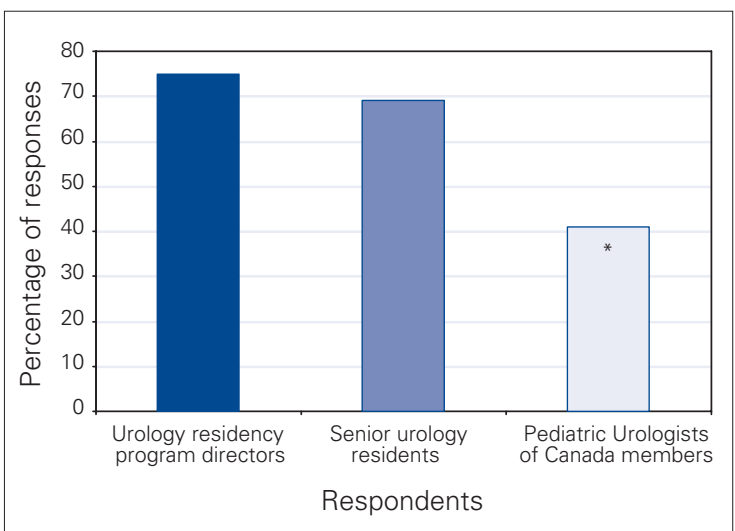

Fig. 1. Percentage of respondents who indicated the amount of time spent in pediatric urology during residency was adequate. ${ }^{*} p=0.05$.

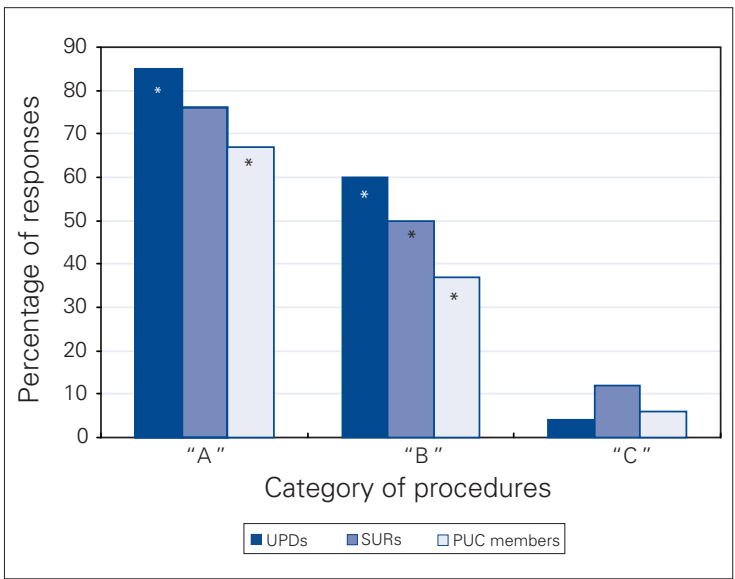

Fig. 2. Percentage of respondents who indicated "yes" for technical competence for each procedure in each category. PUC = Pediatric Urologists of Canada; SURs = senior urology residents; UPDs = urology residency program directors. ${ }^{*} p<0.05$. procedures (Fig. 2). Most of the disagreement surrounded 4 procedures in category " $\mathrm{A}$ " and 3 in category "B" (Fig. 3 and Fig. 4). However, there was unanimity among respondents regarding the actual probability of urology residents performing these various procedures upon graduation (Fig. 5).

\section{Discussion}

Operative competence has been defined as more than merely the technical ability to perform a procedure. It encompasses a much broader domain that captures a trainee's cognitive, technical and communicative skills, as well as confidence, experience,

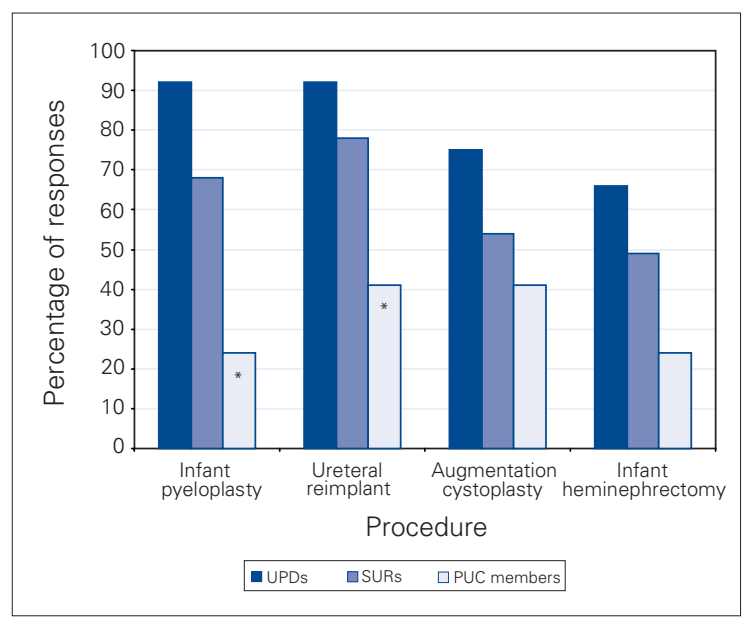

Fig. 3. Percentage of respondents who indicated "yes" for technical competence in controversial procedures from " $\mathrm{A}$ " list accounting for the majority of discrepant responses. PUC = Pediatric Urologists of Canada; SURs = senior urology residents; UPDs = urology residency program directors. $p<0.05$.

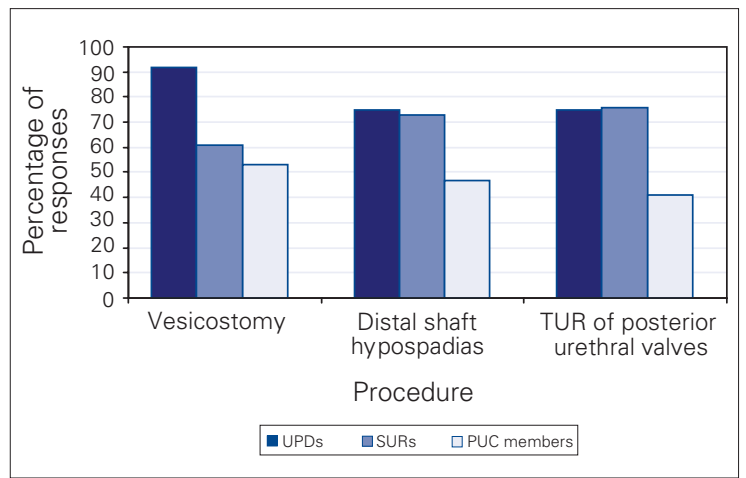

Fig. 4. Percentage of respondents who indicated "yes" for technical competence in controversial procedures from " $\mathrm{B}$ " list accounting for the majority of discrepant responses. PUC = Pediatric Urologists of Canada; SURs = senior urology residents; TUR = transurethral resection; UPDs = urology residency program directors. 
poise, professionalism and judgment. ${ }^{4}$ We surveyed respondents without providing a precise definition of competence, which may partially explain the differing perceptions from the 3 groups. It is also possible that program directors who are not practising pediatric urology do not appreciate the complexity of some of these infant/neonatal procedures. They would, however, be reluctant to acknowledge that their trainees were not competent to perform in areas that are mandatory according to our known Royal College objectives. Similarly, graduating residents may not actually appreciate their level of competence or limitations. In addition, residents may not wish to acknowledge to others that they are not competent in a procedure where competence is deemed mandatory. Resident responses were also based on perceptions at the time of the survey, before actual entry into the workforce. These perceptions may change over time with the realities of actual practice. Conversely, PUC members might be suspected of providing excessively critical responses that stem from a desire for recognition of their extra training and to affirm their subspecialty "turf."

The exact extent to which pediatric urological procedures should be performed by general urologists has not been agreed upon, nor will it likely be in the near future. It has been suggested that a general urologist who understands pediatric urology, and can assume the seamless follow-up of children who have reached puberty, makes a far greater contribution to care than one who performs the occasional complex pediatric procedure. ${ }^{5}$ That said, in Canada, with a relatively sparse population

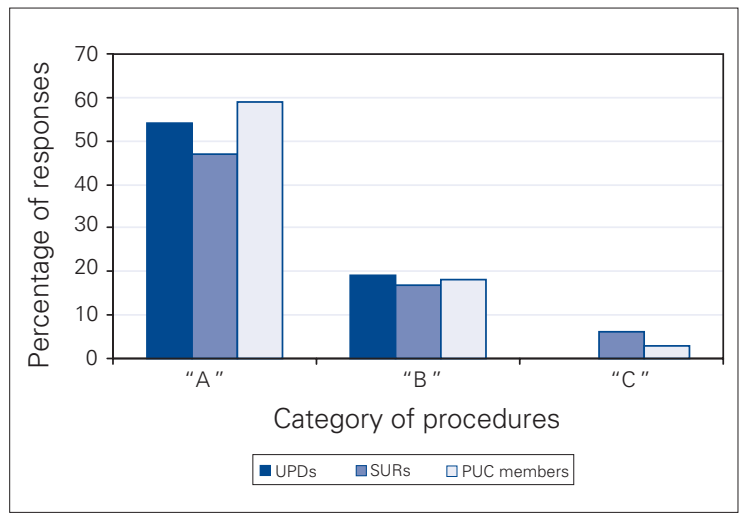

Fig. 5. Percentage of respondents who indicated "yes" for probability of actually performing procedures from each category. PUC = Pediatric Urologists of Canada; SURs = senior urology residents; UPDs = urology residency program directors. dispersed over a large geographic area, it is not practical to expect families to travel great distances to obtain pediatric urological care for all conditions. Consequently many general urologists are required to provide secondary-level care in pediatric urology. This reality was reflected in the results of a recent survey of Canadian urologists: $81 \%$ of respondents indicated that their residency training in pediatric urology was highly useful. ${ }^{6}$ In the United States, the American Board of Urology $(A B U)$ has approved subspecialty certification in pediatric urology. It remains to be seen whether the attainment of a certificate of added qualification in pediatric urology will restrict practice patterns for those without such a designation. However, a review of practice logs submitted to the $\mathrm{ABU}$ for certification or recertification purposes indicates that general urologists (at least in the United States) currently perform almost no major pediatric urological procedures. ${ }^{7}$ Although practice log data do not exist for Canadian urologists, if the situation is similar it may explain our finding that all 3 stakeholders surveyed agreed on the low probability of actually performing most pediatric procedures, regardless of their perceptions of competence.

Similar Canadian assessments of trainee competence have been performed in pediatric general surgery and vascular surgery. ${ }^{8,9}$ In pediatric general surgery there is a long-established process of subspecialty certification with examination sanctioned by the American Board of Surgery in the United States and the RCPSC here in Canada. Despite this formal recognition of pediatric surgery as a separate subspecialty, Canadian general surgery program directors and members of the Canadian Association of Pediatric Surgeons both rated senior resident competence significantly higher than their residents did. Fully $59 \%$ of residents felt inadequately prepared to include pediatric general surgery in their future practice. This finding is at odds with ours in that pediatric urological specialists consistently rated competence lower than program directors and residents. An explanation for this difference between specialties may reside partially with the fact that the average exposure to pediatric general surgery was only 3 months and mostly in the second year of training. In comparison, urology residents averaged 5.4 months in pediatric urology, with all or some of this during the fourth or fifth years of training. The 3 stakeholders in 
the vascular surgery survey mostly agreed on what procedures general surgery residents should and should not be competent to perform without fellowship training. However, $76 \%$ of vascular surgeons felt that the amount of exposure to their subspecialty during general surgery residency was inadequate, similar to our finding that $59 \%$ of PUC members viewed the amount of exposure to pediatric urology as inadequate.

The Objectives of Training in Urology exist as a framework to guide educators and trainees as well as to facilitate the accreditation process for residency programs. They are constructed and revised by consensus among a peer group of urology program directors and educational leaders. It is a fine balance between being too generic versus overly proscriptive when setting objectives, and the current procedural component of these objectives is not an exhaustive list of every conceivable surgical procedure in urology. This allows residency programs the flexibility to provide the subspecialty training that is appropriate for their residents' expected practice type and settings, while still adhering to national standards. As subspecialization evolves within urology, it may become necessary to ensure adequate committee representation from each area of expertise when revising future objectives of training. In the United States, there are only generic requirements applied to all urology training programs, but no specific list of procedures that residents must be competent to perform upon graduation. ${ }^{10}$

There were several limitations to our study. Although the response rates were favourable, our sample sizes were small. In addition, by blinding responses, we were not able to analyze results according to the length and nature of exposure to pediatric urology during residency. It is possible that a resident's perception of competence might differ in a large centre with fellows, compared with the training milieu of a smaller centre in which the resident is the first assistant on all cases. Although this theory merits further exploration, in pediatric general surgery at least, neither the size of a program nor the presence of a fellowship has been found to have any impact on perceived competence. ${ }^{8}$ In addition, we did not query residents on their ultimate intended career path or practice location. This would obviously shape their responses regarding the probabilities of performing various procedures. Finally, the addition of a sampling of recently graduated urologists to the survey would have added a real-life dimension to help confirm the validity of the responses from the other 3 groups.

Several questions arise from our findings that may merit further study. For example, why in particular do UPDs and PUC members disagree on trainee competence with respect to infant pyeloplasty and ureteral reimplantations? Is there a lack of communication between PUC members who are teaching faculty and their respective program directors that explains their discrepant perceptions? If PUC members and residents both agree that urology residents are not competent in some of the " $\mathrm{A}$ " list procedures, does this infer that more time should be spent on pediatric urology rotations, or should the procedures in question be placed in the " $\mathrm{B}$ " or " $\mathrm{C}$ " list instead?

\section{Conclusion}

This study provides useful information on the extent and efficacy of pediatric urological training in Canadian urology residency programs. It should serve as an initial investigation into the current Canadian experience. The data reported are based on perceptions. Like all perceptions, they are subject to personal and professional biases, which are difficult to assess. Further investigation regarding exposure to and competence in other emerging subspecialty spheres of urology (e.g., minimally invasive surgery/robotics, female pelvic medicine and reconstructive surgery, oncology) may be warranted. As always, our objectives of training in urology need to be reassessed on an ongoing basis.

From the *Department of Pediatric Urology, Children's Memorial Hospital, Chicago, III., the †Department of Pediatric Urology, BC Children's Hospital, Vancouver, BC, the ‡Department of Urological Sciences, University of British Columbia, Vancouver, $B C$, and the §Department of Urology, Queen's University, Kingston, Ont.

This article has been peer reviewed.

Competing interests: None declared.

\section{References}

1. Rushton HG. Subspecialty cerrification in pediatric urology. J Urol 2006;173:1845-6.

2. The Society for Pediatric Urology. Pediatric subspecialty certification. Available: www.spuonline.org/Pediatric-Subspecialty-Certification/ (accessed 2007 July 9).

3. The Royal College of Physicians and Surgeons of Canada. Objectives of training in urology. Available: http://repsc.medical.org/information/index?specialty=360\& submit=Select (accessed 2007 July 9). 
Mickelson et al.

4. Grober ED, Jewett MAS. The concept and trajectory of "operative competence" in surgical training. Can I Surg 2006;49:239-40.

5. Woodhouse CRJ. Which pediatric urological conditions can be handled by adult urologists? Nature clinical practice. Urology 2005;2:152-3.

6. Morrison KB, MacNeily AE. Core competencies in surgery: assessing the goals of urology residency training in Canada. Can I Surg 2006;49:259-66.

7. Howards SS. Subspecialty certification in pediatric urology. ABU Report 2006;1(14). Available: www.abu.org/ABUSearch/downloads/2006Newsletter.pdf (accessed 2008 Apr 11).

8. Poenaru D, Fitzgerald P. Training general surgery residents in pediatric surgery: a Canadian survey. J Pediatr Surg 2001;36:706-10.

9. Sidhu $R S, K_{0} M$, Rotstein $L$, et al. Vascular surgery training in general surgery residency programs: the Canadian experience. J Vasc Surg 2003;38:1012-7.

10. Accreditation Council for Graduate Medical Education. Urology program requirements. Available: www.acgme.org/acWebsite/RRC_480/480_prlndex.asp (accessed 2007 July 11).

Correspondence: Dr. Andrew E. MacNeily, K0-134 BC Children's Hospital, 4480 Oak St., Vancouver BC V6H 3V4; amacneily@cw.bc.ca

\section{Appendix 1: Pediatric urology competency - survey for Canadian senior residents}

Dear senior urology resident, thank you for completing the survey below.
Level of training:
$\mathrm{Rl}$
RII RIII
RIV
RV

Number of months of pediatric urology as a junior resident (RI-II):

Number of months of pediatric urology as a senior resident (RIII-V):

Total number of months of pediatric urology training during your residency:

Overall, how do you feel about the amount of pediatric urologic training in urology? (too little, just right, too much)

Please indicate which procedures you feel technically competent to perform and which procedures you will perform in your career as a urologist (without further training):

Technically competent Will perform in practice

Procedure

Yes

No

Yes

No

Circumcision

Incarcerated hernia

Exploration of testicular torsion

Infant hernia/hydrocele repair

Palpable orchiopexy

Nonpalpable orchiopexy

Laparoscopy for nonpalpable testis

Proximal hypospadias repair

Distal shaft hypospadias repair

Meatoplasty

Meatal repair for balanic hypospadias

Cutaneous ureterostomy

Exstrophy repair

Infant pyeloplasty

Infant heminephrectomy

Ureteral reimplant

Vesicostomy

Transurethral resection of posterior

urethral valves

Augmentation cystoplasty

Pediatric continent diversion

Insertion of suprapubic tube

Renal transplant

Endourologic procedures for urolithiasis

Additional comments: 